\title{
INTS8 Predicts a Therapeutic Target for Intrahepatic Cholangiocarcinoma via Integration of Bioinformation Analysis and Experimental Validation
}

\section{Qi Zhou}

The First Affiliated Hospital of Dalian Medical University

\section{Li Ji}

Liaoning University of Traditional Chinese Medicine

\section{Xueying Shi}

The First Affiliated Hospital of Dalian Medical University

\section{Dawei Deng}

The First Affiliated Hospital of Dalian Medical University

\section{Fangyue Guo}

The First Affiliated Hospital of Dalian Medical University

\section{Zhengpeng Wang}

Dalian Medical University

Wenhui Liu

Dalian Medical University

Jinnan Zhang

Dalian Medical University

Shilin Xia ( $\nabla$ xiashilin@dmu.edu.cn)

The First Affiliated Hospital of Dalian Medical University

\section{Dong Shang}

The First Affiliated Hospital of Dalian Medical University

\section{Research Article}

Keywords: Intrahepatic cholangiocarcinoma, INTS8, immune infiltration, pan-cancer analysis

Posted Date: May 20th, 2021

DOl: https://doi.org/10.21203/rs.3.rs-523277/v1

License: (c) (i) This work is licensed under a Creative Commons Attribution 4.0 International License. 



\title{
INTS8 Predicts a Therapeutic Target for Intrahepatic Cholangiocarcinoma via Integration of Bioinformation Analysis and Experimental Validation
}

\author{
Qi Zhou ${ }^{1,2 \#}$, Li Ji ${ }^{3 \#}$, Xueying $\mathrm{Shi}^{1,2 \#}$, Dawei Deng ${ }^{4}$, Fangyue Guo ${ }^{1,2}$, Zhengpeng \\ Wang ${ }^{2}$, Wenhui Liu ${ }^{2}$, Jinnan Zhang ${ }^{2}$, Shilin $\mathrm{Xia}^{1 *}$, and Dong Shang ${ }^{1,2,4^{*}}$ \\ ${ }^{1}$ Clinical Laboratory of Integrative Medicine, The First Affiliated Hospital of Dalian \\ Medical University, No.222 Zhongshan Road, Dalian, China. \\ ${ }^{2}$ Institute (College) of Integrative Medicine, Dalian Medical University, No.9 West \\ Section Lvshun South Road, Dalian, China.
}

3 Department of Gastroenterology, Liaoning University of Traditional Chinese Medicine, No.79 Chongshandong Road, Shenyang, China.

${ }^{4}$ Department of General Surgery, The First Affiliated Hospital of Dalian Medical University, No.222 Zhongshan Road, Dalian, China.

*Corresponding author,

Prof. Shilin Xia

Clinical Laboratory of Integrative Medicine, The First Affiliated Hospital of Dalian Medical University, No.222 Zhongshan Road, Dalian, China.

E-mail: xiashilin@dmu.edu.cn

Prof. Dong Shang

Department of General Surgery, The First Affiliated Hospital of Dalian Medical University, No.222 Zhongshan Road, Dalian, China.

Tel: $+86-411-83635963$

Fax: +86-411-83622844

E-mail: shangdong@dmu.edu.cn 
\# These authors contribute equally as first authors.

* These authors contribute equally as corresponding authors.

\begin{abstract}
Background \& Aims: Intrahepatic cholangiocarcinoma (ICC) remains a rare malignancy, ranking the leading lethal primary liver cancer worldwide. However, biological functions of integrator complex subunit 8 (INTS8) remain unknown in ICC. Thus, this research aimed to explore the potential role of INTS8, as well as develop a novel diagnosed or therapeutic target in ICC. Methods: Differently expressed genes in two GEO datasets were obtained by RRA package in R software. The "maftools" package was implemented to visualize the cholangiocarcinoma (CHOL) mutation data in TCGA database. The expression of INTS8 was detected by preforming quantitative reverse transcription-PCR (qRT-PCR) and immunohistochemistry in cell lines and human samples, respectively. The association between subtypes of tumor-infiltrating immune cells (TICs) and INTS8 expression in CHOL was performed by using CIBERSORT tools. We evaluated a correlation between INTS8 expression and mismatch repair genes (MMRs) and DNA methyltransferases in pan-cancer analyses. Finally, the pan-cancer prognostic signatures of INTS8 were identified by univariate analyses. Results: We obtained the mutation landscape of a RRA gene set in CHOL. The expression of INTS8 was actually upregulated in ICC cell lines and human ICC samples. Furthermore, INTS8 expression is tightly associated with distinct landscape of TICs, the MMRs, and DNA methyltransferases in CHOL. In addition, the high INTS8 expression group presented a significantly poor outcomes, including overall survival (OS), disease-specific survival (DSS) and disease-free interval (DFI) $(P<0.05)$ in multi-cancers. Conclusions: INTS 8 contributes to the tumorigenesis and progression of ICC. Our study highlights the significant roles of INTS8 in ICC and pan-cancers, providing a valuable molecular target for cancer research.
\end{abstract}


Keywords: Intrahepatic cholangiocarcinoma; INTS8; immune infiltration; pan-cancer analysis

\section{Introduction}

As one of the hepatobiliary malignancy subtypes, intrahepatic cholangiocarcinoma (ICC) has attracted more attentions due to its increasing global incidence and trends ${ }^{[1]}$, with the largest age-standardized incidence rates increased in China (average annual percent change: $11.1 \%$ ) for ICC from 1993 to $2012^{[2]}$. Moreover, the mortality rate from ICC showed a rising trend globally, which was around 1-2/100,000 in most countries ${ }^{[3]}$. Attributing to the aggressive and asymptomatic features of ICC, many patients are diagnosed at an advanced stage. Surgical resection is regarded as the best treatment strategy for achieving a good prognosis and long survival. However, a 5-year overall survival rate remains limited to $22 \%-30 \%$ after curative hepatectomy due to high recurrence rates ${ }^{[4-5]}$. Moreover, $40-80 \%$ recurrence rate was reported for ICC patients after surgical resection ${ }^{[6]}$. The combination of gemcitabine and cisplatin was regarded as the standard- chemotherapy regimen, despite showing limited effectiveness for ICC. Therefore, it is urgent to improve the sensitivity of diagnose and effectiveness of targeted therapy for ICC.

Integrator is reported as a transcriptional regulatory complex, which is comprised by at least 14 subunits ${ }^{[7]}$. Integrator complex subunit 8 (INTS8) serves as one of the major components of RNA polymerase II, has been demonstrated to involve in the cleavage of small nuclear RNAs and transcriptional processes ${ }^{[8-9]}$. A recent study found that INTS8 was essential to transcription repression, which was induced by recruiting protein phosphatase $2 \mathrm{~A}$ to prevent transcription elongation and promote transcription termination ${ }^{[10]}$. Previous study revealed that the INTS8 was robustly increased in neurodevelopmental diseases ${ }^{[11]}$ and numerous tumors ${ }^{[12-13]}$. Overexpressed INTS8 could facilitate the epithelial-to-mesenchymal transition, which was mediated by TGF$\beta$ signaling pathway in hepatocellular carcinoma ${ }^{[14]}$. However, studies concentrated on the effect of INTS8 in ICC are generally lacking. 
In the present study, we performed robust rank aggregation (RRA) method to select differently expressed genes (DEGs) based on Gene Expression Omni (GEO) database. Then, we explored genes at an intersection between DEGs and gene mutation profiles in cholangiocarcinoma cohort (CHOL) of the cancer genome atlas (TCGA), and identified INTS8 as a candidate gene. We verified the overexpression of INTS8 in ICC cell lines and human ICC samples by quantitative reverse transcription-PCR (qRTPCR) and immunohistochemistry (IHC), respectively. Our study showed that the high INTS8 expression has a tight correlation with poor prognosis in multi-cancers. Moreover, the underlying mechanism may attribute to tumor-infiltrating immune cells (TICs), the mismatch repair genes (MMRs), and DNA methyltransferases. Therefore, INTS8 was identified to be a therapeutic target for in ICC and pan-caner, which was related to the TICs, and mediated by MMRs and DNA methyltransferases.

\section{Materials and Methods}

\subsection{Data Acquisition and Processing}

We selected 2 ICC datasets from Gene Expression Omnibus (GEO) (http://www.ncbi.nlm.nih.gov/geo/), including GSE26566 and GSE32225 [15-16]. GSE26566 included 104 cholangiocarcinoma samples and 6 matched surrounding samples. While GSE32225 contained 149 cholangiocarcinoma samples and 6 matched surrounding samples. All expression profiles were downloaded and processed by "GEOquery" package (www.r-project.org). Considering the differences and batch effects of different platforms, we utilized the "sva" package ${ }^{[17]}$ to avoid these effects and remove other unwanted variation. In addition, transcriptome profiling, mutation and methylation data, as well as clinical information of tumor samples and corresponding samples were obtained from the TCGA database (https://cancergenome.nih.gov/), which were analyzed by utilizing the "TCGA biolinks" package ${ }^{[18]}$. Transcripts per million (TPM) values were applied for subsequent analyses [19]. 


\subsection{DEGs and RRA Analysis}

The DEGs were determined between cholangiocarcinoma samples and matched surrounding samples by using the "limma" package ${ }^{[20]}$. Robust rank aggregation was performed for gene list integration ${ }^{[21]}$, and the score $<0.05$ was regarded to determine RRA gene set. These data were visualized by heatmap and volcano plot.

\subsection{Identification of INTS8 by mutation analysis and ROC curves}

We administrated the "maftools" package ${ }^{[22]}$ to visualize the CHOL mutation data. To assess mutated genes presented in RRA gene set, we obtained the intersection of RRA genes and CHOL mutation genes. To evaluate the diagnostic performance of muted RRA genes, ROC curves was performed by using pROC. Next, we selected optimal efficacy indicator for the further research according to the areas under the curve (AUCs). The patients were stratified into two groups according to the median expression of INTS8. DEGs between high/low INTS8 groups were confirmed by using "Limma" package. The cutoffs for the DEGs were as follows: $|\log 2 \mathrm{FC}|>1$, and false discovery rate $(\mathrm{FDR})<0.05$. A heat map by using ggplot 2 packages was used to visualize the DEGs.

\subsection{Functional enrichment of mutated RRA genes and INTS8 related genes}

To identify possible pathways and biological functions of 5 mutated RRA genes and differential INTS8-associated genes, we applied the "clusterProfiler" package ${ }^{[23]}$ to perform Gene Ontology (GO) and Kyoto Encyclopedia of Genes and Genomes (KEGG). The PPI network of 5 mutated RRA genes was constructed via STRING (http://string-db.org/) online database, and then visualized by Cytoscape v.3.7.1 (https://cytoscape.org/). Moreover, we used the Molecular Complex Detection (MCODE) to explore functional clusters in PPI network. In order to further understand the INTS8-related function and biological pathways, Gene Set Enrichment Analysis (GSEA) analysis was done on the gene INTS8 by utilizing GSEA software (v.3.0). 


\subsection{CIBERSORT estimation}

In order to characterize the tumor microenvironment, we applied "CIBERSORT" (R package) (http://cibersort.stanford.edu/) ${ }^{[24]}$ to explore the relative proportions and absolute fraction score of 22 subtypes of TIICs in CHOL tissues. Moreover, the association between the INTS8 expression levels and the infiltration of TIICs was also assessed and visualized by heatmaps and violin plots.

\subsection{The association of INTS8 gene expression with clinical outcome in different tumors}

To explore the influence of expression level of INTS8, we carried out analyses by publicly available database. we retrieved data from The Cancer Cell Line Encyclopedia (CCLE, https://portals.broadinstitute.org/ccle $)^{[25]}$ and The Genotype-Tissue Expression $(\mathrm{GTEx})^{[26]}$ for investigating gene expression data of INTS8 in a range of tumor tissues and cell lines. Moreover, we downloaded pan-cancer mutation data from the TCGA database, and analyzed the mutations of INTS8 in each tumor sample among 32 different tumor types. Furthermore, we used the Cox regression and the Kaplan-Meier method to evaluate the association of INTS8 gene expression with clinical outcome in different cancer tumors. $P<0.05$ was regarded as the cutoff to verify the prognostic role of INTS8.

\subsection{The expression of INTS8 associated with both mismatch repair genes and DNA} methylation

To reveal the role of INTS8 in cancer progression, we evaluated the relationship between expression level of INTS8 and 5 key DNA MMRs (including MLH1, MSH2, MSH6, PMS2, EPCAM). In addition, we constructed an integrative analysis of DNA methylation and INTS8 expression to determine its underlying mechanism in pancancer.

\subsection{Real-time PCR}


A human normal biliary epithelial cell line (HIBE) and 3 ICC cell lines (including HCCC-9810, RBE, and CCLP-1 cells) were used to detect the mRNA expression of INTS8. Total RNA and cDNA synthesis were performed by following the manufacturer's instructions (Accurate biotechnology, China). Gene expression was measured on an ABI 7500 by using SYBR Green Kit (Accurate biotechnology, China). The forward primer for INTS8 was 5'-TGCTGGAGGAGTCACTGTTGGAG- 3', the reverse primer for INTS8 was 5'-TTATCAGGCGGAGGTTGAACTTGG-3'.

\subsection{Immunohistochemistry}

A total of 155 paired ICC and 5 peritumoral tissue samples were obtained for the experimental validation. Informed consent was obtained from all participants. The study involving human participants was approved by the Ethics Committee of Shanghai Outdo Biotech Company with NO. YB M-05-02, and performed following relevant guidelines and regulations. The formalin-fixed paraffin-embedded tissue samples were examined by incubating with primary antibodies (ab18050, abcam).

\section{Results}

\subsection{The identification of the robust DEGs in GEO}

Based on the results of DEGs, a total of 710 up-regulated and 903 down-regulated significant DEGs were confirmed in GSE26566, and 432 up-regulated and 566 downregulated significant DEGs were found in GSE32225. The DEGs were shown by heatmaps and volcano plots (Figure 1A-D). Furthermore, these DEGs were integrated by RRA method with the score $<0.05$. And then RRA gene set was visualized by a heatmap (Figure 1E). In a result, a RRA gene set was obtained for further investigation.

\subsection{Functional enrichment and PPI network analysis of the RRA gene set}

GO and KEGG enrichment analyses displayed the functions of the RRA gene set (Supplementary Figure 1A-B). The RRA gene set was obviously enriched in biological processes, such as lipid catabolic process, digestion, drug catabolic process, and 
icosanoid metabolic process. Besides, the RRA gene set also participated in pancreatic secretion, fast digestion and absorption, protein digestion and absorption, and focal adhesion (Supplementary Figure 1C-D). A PPI network of RRA gene set was constructed to identify protein interactions, which included 202 interactions, and visualized by Cytoscape (Supplementary Figure 2A, 2C). Two functional clusters in PPI network were extracted, suggesting their central roles in this network (Supplementary Figure 2B). Our result showed that the RRA gene set was associated with some metabolic pathways.

\subsection{Mutation landscape of RRA gene set in CHOL}

To identify mutational landscape in CHOL patients, the "maftools" package in $\mathrm{R}$ software was implemented. Missense mutations were the predominate type of mutation in patients with CHOL (Figure 2A). Single nucleotide polymorphism had a more frequent occurrence compared with insertion or deletion (Figure 2B). Particularly, C > T remained the most common mutation type of single nucleotide variants in $\mathrm{CHOL}$ (Figure 2C). Moreover, the mutation types were displayed for CHOL (Figure 2D-E). The top 10 mutated genes were presented in CHOL with ranked percentages, including MUC16 (12\%), PBRM1 (20\%), ARID1A (18\%), BAP1 (16\%), MUC5B (10\%), EPHA2 (14\%), IDH1 (12\%), LRP1B (10\%), CHD7(10\%), and DNAH5 (8\%) (Figure 2F). A total of 5 mutation genes of RRA gene set were found in mutation profiles, and the mutation information of RRA gene set was performed by the waterfall plot (Figure 2G). The top mutant genes of RRA gene set showed that BAP1, IDH1 and PBRM1 were the top 3 mutant genes (Figure $2 \mathrm{H}$ ). The mutant base pair ratio of RRA gene set displayed that $\mathrm{C}>\mathrm{T}$ was the most common mutation type of single nucleotide variants in RRA gene set (Figure 2I).

\subsection{Identification of DEGs between high and low INTS8 expression groups}

The ROC analysis was applied to determine the diagnostic efficacy of the 5 mutated genes from RRA gene set. Moreover, the INTS8 had the highest AUC value $(\mathrm{AUC}=0.852)$, followed by ATF4 $(\mathrm{AUC}=0.836)$, PPP1CA $(\mathrm{AUC}=0.781)$, PCSK2 
$(\mathrm{AUC}=0.504)$ and BUB1B $(\mathrm{AUC}=0.5)$, respectively (Figure 3A). Due to the highest AUC performance of INTS8, INTS8 was selected as the target gene for further analysis. To explore the underlying mechanism of INTS8 in ICC, patients were divided into two groups according to median expression value of INTS8. DEGs were displayed between high and low INTS8 expression groups (Figure 3B). Furthermore, we found the mRNA expression of INTS 8 was actually upregulated in 3 ICC cell lines compared with HIBEC in vitro (Figure 3C). Meanwhile, the protein levels of INTS8 by using IHC were also verified to be obviously increased in ICC patients tissue compared with the normal samples (Figure 3D). The experimental results were consistent with that of the bioinformatic analysis.

\subsection{Functional enrichment of INTS8 in CHOL}

To identify the biological function and key candidate pathways of the INTS8 related genes, we performed GO and KEGG analysis. As shown in Figure 4A, the top $10 \mathrm{GO}$ terms were exhibited. Drug metabolism-cytochrome P450, retinol metabolism, chemical carcinogenesis, metabolism of xenobiotics by cytochrome P450, drug metabolism-other enzymes, and fatty acis degradation were the most significantly enriched in CHOL patients with high INTS8 expression than low INTS8 expression group (Figure 4B). In order to elucidate the molecular mechanisms of the INTS8, INTS8-related signaling pathways were analyzed by GSEA-KEGG and GSEA-GO (Figure 4C-D). The results suggested that INTS8 might be related to metabolic pathways, such as cytochrome P450 and retinol metabolism.

\subsection{Association between tumor-infiltrating immune cells and INTS8 expression in CHOL}

TIICs significantly impact the development and progression of many types of cancers, including CHOL. By applying CIBERSORT tools, we observed a high level of M0 macrophages, M2 macrophages, Monocytes, and resting CD4+ memory T cells, while a lower level of activated Dendritic cells, Eosinophils, Neutrophils and activated CD4+ memory $\mathrm{T}$ cells in CHOL (Figure 5A-B). Moreover, we assessed the relationship 
between TIIC and INTS8 expression in CHOL. We found that high INTS8 expression group presented a unique TIICs landscape, including statistically significantly high level of M0 macrophages but low level of M2 macrophages, elevated resting CD4+ memory $\mathrm{T}$ cells but low level of CD4 native T cells, increased resting mast cell but low activated mast cell. In addition, low expression of gamma delta T cells and monocytes were also found in high INTS8 expression group (Figure 5C-D).

\subsection{The INTS8 expression in multiple dimensions}

Considering the extensive mutational heterogeneity of cancers, we systematically performed the large-scale profiling of INTS8 expression in 21 cell lines and 31 related tissues based on CCLE and GTEx. As shown in Figure 6A-B, the expression levels of INTS8 in diverse cancer tissues and cell lines were elevated to differing degrees, including biliary tract, liver, bone marrow. In addition, we also found INTS8 harbored most prevalent mutations such as missense, truncating and fusion in different tumors (Figure 6C).

\subsection{The association between INTS8 and clinicopathologic characteristics and survival information}

As show in Table 1, increased INTS8 expression was directly associated with the age, and grade. The INTS8 expression was gradually increased in stages I and II ICC. To assess the prognostic capacity of INTS8, we performed Kaplan-Meier curve for OS, DSS, and DFI by using multivariate Cox regression analysis. In prognosis outcomes, patients in the high INTS8 group exhibited evident poor OS, DSS and DFI $(\mathrm{P}<0.05)$ in a pan-cancer analysis (Supplementary Figure 3-5). These findings suggested that INTS8 expression showed the potent potential prognostic biomarker for pan-cancers.

\subsection{The MMRs and DNA methylation involved in CHOL}

Increasing evidence has demonstrated that MMRs play an important role in maintaining genomic stability, and DNA methylation regulates gene expression. The loss of key 
gene function of MMR could induce DNA replication errors, resulting in high level of somatic mutations. It has been reported that MMR pathway was potently activated during G1/S-phase ${ }^{[27]}$. To explore the underlying DNA repair associated with INTS8 mutation, we investigated an association between INTS8 and MMRs (including MLH1, MSH2, MSH6, PMS2, and EPCAM). We found that INTS8 was positively correlated with the expression of MSH2, MSH6, PMS2, but showed no association with MLH1 and EPCAM. Due to the extensive function of MMRs in cancers, we performed a pancancer analysis to analyze the relation between INTS8 and MMRs. Interestingly, positive association between INTS8 and MMRs was present in numerous cancers, such as brain lower grade glioma, liver hepatocellular carcinoma, and pancreatic cancer (Figure 7A).

DNA methylation serves as an epigenetic modification, which could regulate gene expression. Attributed to the function of DNA methyltransferase, DNA methylation was processed when the covalent bonding of a methyl group to the 5 'carbon position of the cytosine in genome $\mathrm{CpG}$ dinucleotide ${ }^{[28]}$. As shown in Figure $7 \mathrm{~B}$, an epigenetic signature was discovered, and showed a high correlation between INTS8 and DNA methyltransferase (DNMT1: $\mathrm{r}=0.31, \mathrm{p}<0.05$; DNMT2: $\mathrm{r}=0.53, \mathrm{p}<0.05$; DNMT3A: $\mathrm{r}=0.53, \mathrm{p}<0.05 ;$ DNMT3B : $\mathrm{r}=0.42, \mathrm{p}<0.05)$. Furthermore, a pan-cancer analysis of DNA methyltransferase was effectively stratified, showing that INTS8 was positively related to the expression profiles of 4 DNA methyltransferase in most cancers except in testicular germ cell tumor (TGCT). All those results indicated that that MMRs and significant DNA methyltransferase may play a potent role in the INTS8 mutation for CHOL.

\section{Discussion}

ICC is an extremely aggressive biliary neoplasm with increasing incidence and poor prognosis worldwide ${ }^{[29]}$. The current diagnostic and prognostic marker for ICC remained unsatisfactory. In this study, TCGA and GEO database were applied to systematically analyze the mutational status of RRA genes in ICC, and 5 mutant genes were found by intersection analysis. Based on the diagnosis efficacy of the 5 mutant 
genes, we selected the INTS 8 with the largest AUC value for follow-up research, which showed a significant role in ICC even across pan-cancer.

Various evidence suggested that the integrator complex played an essential role in the aspect of RNA processing and transcription regulation. Previous studies have showed that INTS8 mutation could induce a severe neurodevelopmental syndrome ${ }^{[11]}$, and multi-cancers ${ }^{[30]}$. In this study, we described that INTS8 was significantly overexpressed in ICC compared to normal samples, which was consistent with the results of IHC and PCR. Our results showed that overexpressed INTS 8 was positively related to the poor prognosis in many tumor types.

The GO enrichment analyses showed that high INTS8 expression was mainly associated with organic anion transport, organic acid transport, carboxylic acid transport and acute inflammation response. In addition, retinol metabolism, chemical carcinogenesis, drug metabolism-cytochrome P450, metabolism of xenobiotics, drug metabolism-other enzymes, fatty acids degradation were most significantly enriched in CHOL patients with high INTS8 expression than low INTS8 expression group. Retinol belongs to fat-soluble nutrients, which is essential to maintain physiological functions in many tissues [31]. Retinol metabolism abnormality caused by genetic or environmental factors could induce developmental pathologies, including mammalian placental and embryonic development ${ }^{[32]}$, ovary disease ${ }^{[31]}$ and fatty liver disease ${ }^{[33]}$. Previous study showed that the administration of retinol facilitated hepatocarcinogenesis development during its early stages ${ }^{[34]}$. Cytochrome P450 had a vital role in mediating hepatic metabolism, especially its clinical significance in the metabolism of some opioid and hypnotic drugs ${ }^{[35]}$. Drug metabolism-cytochrome P450 was related to DNA methylation-driven genes in prostate adenocarcinoma ${ }^{[36]}$. In addition, previous data showed that hepatic cytochrome P450 (CYP) family enzymes, especially increased CYP2A6 and diminished CYP2E1 might participate the progression of ICC ${ }^{[37]}$. Lipid metabolism is newly recognized as a hallmark of cancer, and inhibiting fatty acid availability could control the development of malignancy ${ }^{[38-}$ 39]. Li et al. found that ICC tumorigenesis was insensitive to FASN deprivation, which contributed to high expression of fatty acid uptake and resulted in rapid tumor growth. 
Therefore, promoting fatty acid degradation may be a novel therapeutic approach for the ICC treatment options ${ }^{[40]}$.

DNA damage and repair provide protection for mutation avoidance, which play central roles in maintaining genome stability ${ }^{[41-42]}$. Up to date, it has been reported that 4 major DNA repair pathways involved in maintaining the gene expression, including nucleotide excision repair, base excision repair, MMR, and double strand break repair ${ }^{[43]}$. To estimate an association of INTS8 and MMRs, we assayed an association of INTS8 and MSH2, MSH6, PMS2, and EPCAM. The expression of INTS8 was positively correlated to MSH2, MSH6, PMS2, but not associated with MLH1 and EPCAM. By using the immunohistochemical analysis ${ }^{[44]}$, the results showed that there is no loss of the expression of DNA repair enzymes/MMR proteins (MLH1, MSH2, PMS2, and MSH6) in both Occupational cholangiocarcinoma (CCA) ${ }^{[45]}$ and cohorts with cholangiocarcinoma ${ }^{[46]}$.

MMR gene mutations or tumor MLH1 promoter methylation was concerned as a main cause of microsatellite instability (MSI) in patients with Colorectal cancer (CRC) ${ }^{[47]}$. After analyzing 96 cases of intra- and extrahepatic cholangiocarcinomas for MMR status by using IHC, Jennifer Y Ju et al. found that $6 \%$ of samples presented with MMR deficiency ${ }^{[48]}$. Although microsatellite instability relatively frequently occurred in in intraductal papillary neoplasms of the biliary tract, the methylation of hMLH1 was not detected ${ }^{[49]}$. EpCAM, a stemness-related marker, is positively with the aggressive prognosis in ICC and HCC ${ }^{[50-51]}$. However, we didn't observe an association between INTS8 and EpCAM in ICC.

Recently, Epigenetic alterations is characterized by any heritable modification of chromatin DNA or histone proteins but without change in the DNA sequence ${ }^{[52-53]}$, which can be observed in many human cancers, and cooperate with genetic alterations to dominate the formation of cancers ${ }^{[54]}$. DNA methylation is one of the main presence of epigenetic changes, which is specifically mediated by the DNA methyltransferase (DNMT) family (including DNMT1, DNMT1, DNMT3A and DNMT3B) ${ }^{[55]}$. DNMTs could establish and maintain DNA methylation patterns, which induced gene silencing, transcriptional activation and post-transcriptional regulation mediated by DNMT2- 
dependent RNA methylation. Here, we found that INTS8 is positively associated with DNMTs in CHOL, suggesting that INTS8 attributing to ICC development may be caused by mutations in DNA methyltransferase genes. Thus, we hypothesized that functional impairment of INTS8 promote malignancies, which is associated with MMRs and the DNA methyltransferase in pan-cancers, suggesting the potential of INTS8 for cancer research.

A significant increase of macrophage was showed in locally advanced CCA patients compared to the metastatic CCA ${ }^{[56]}$. In our study, an immune landscape showed a distinct high expression of macrophages in ICC, which was consistent with other studies. However, the result demonstrated that low M2 macrophages level but no significant alteration of M1 macrophages appeared in high INTS8 expression group, suggesting remarkable cellular heterogeneity exists in macrophages subtypes. M1 macrophages is currently known for promoting inflammation, but M2 macrophages are characterized by anti-inflammation function. Thus, whether the INTS 8 present a locally advanced ICC remain more experimental validation. Particularly, a case study has discovered the $\gamma \delta$ T cell-based immunotherapy showed no adverse effects, and could positively regulated peripheral immune functions for the patient with ICC ${ }^{[57]}$. we identified low gamma delta T cells level was present in high INTS8 expression group in ICC. Based on this promising finding, INTS8 could be developed into a promising therapy for ICC. Previous studies proved that CD4 regulatory T cells is prominent immunosuppressive characteristics in ICC ${ }^{[58]}$. Although Tregs was relatively high in ICC, but had no association with INTS8.

Although our study improved insights into the relationship between INTS8 and ICC, there were still some limitations. Due to the smaller cases occurrence compared with other common cancers, the samples size for IHC involved in cohort validation (especially for the peritumoral tissue samples) was relatively small. In addition, the samples with complete clinical information from TCGA were relatively few. However, in order to avoid a bias caused by the small sample size, we used GEO and TCGA data to lend support for each other, and finally extended the potential functions and 
mechanisms of INTS 8 to pan-cancer research, and discussed the functions of INTS8 in depth. Despite the association between INTS8 expression with MMR and DNA methyltransferases was showed, the direct mechanisms require a further exploration by solid evidence from experiments.

\section{Conclusion}

In summary, we observed increased INTS8 expression can contribute to malignancies, which was directly associated with age, grade, sex in ICC. Moreover, a pan-cancer analysis revealed that alter expression of INTS8 might participate in the development of multiple cancer types, which may be mediated by MMRs and DNA methylation status. In addition, the high INTS8 group displayed an obvious poor prognosis in OS, DSS, and DFI in multi-cancer types. Our results predicted the potential of INTS8 as a therapeutic target for ICC.

\section{Abbreviations}

AUCs: areas under the curve; BER: base excision repair; CCLE: the Cancer Cell Line Encyclopedia; CHOL: cholangiocarcinoma; CRC: colorectal cancer; CYP: cytochrome P450; DEGs: differently expressed genes; DFI: disease-free interval; DSBR: double strand break repair; DSS: disease-specific survival; FDR: false discovery rate; GEO: Gene Expression Omni; GO: Gene Ontology; GSEA: Gene set enrichment analysis; GTEx: the Genotype-Tissue Expression; HCC: hepatocellular carcinoma; HIBE: human normal biliary epithelial cell line; ICC: intrahepatic cholangiocarcinoma; IHC: immunohistochemistry; INTS8: integrator complex subunit 8; KEGG: Kyoto Encyclopedia of Genes and Genomes; MCODE: Molecular Complex Detection; MMRs: mismatch repair genes; OS: overall survival; RRA: robust rank aggregation; STRING: Search Tool for the Retrieval of Interacting Genes; TCGA: the cancer genome atlas; TGCT: testicular germ cell tumor; TICs: tumor-infiltrating immune cells.

\section{Reference}

1 Rizvi S, Khan SA, Hallemeier CL, Kelley RK, and Gores GJ. 
Cholangiocarcinoma - evolving concepts and therapeutic strategies. Nat Rev Clin Oncol. (2018) 15:95-111. doi:10.1038/nrclinonc.2017.157

2 Florio AA, Ferlay J, Znaor A, Ruggieri D, Alvarez CS, Laversanne M, et al. Global trends in intrahepatic and extrahepatic cholangiocarcinoma incidence from 1993 to 2012. Cancer. (2020) 126:2666-78. doi:10.1002/cncr.32803

3 Bertuccio P, Malvezzi M, Carioli G, Hashim D, Boffetta P, El-Serag HB, et al. Global trends in mortality from intrahepatic and extrahepatic $\begin{array}{llll}\text { cholangiocarcinoma. } & J & \text { Hepatol. } & \text { (2019) }\end{array}$ doi:10.1016/j.jhep.2019.03.013

4 Chun YS, and Javle M. Systemic and Adjuvant Therapies for Intrahepatic Cholangiocarcinoma. Cancer Control. (2017) 24:1073274817729241. doi:10.1177/1073274817729241

5 Miyata T, Yamashita YI, Yoshizumi T, Shiraishi M, Ohta M, Eguchi S, et al. CXCL12 expression in intrahepatic cholangiocarcinoma is associated with metastasis and poor prognosis. Cancer Sci. (2019) 110:3197-203. doi:10.1111/cas.14151

6 Sirica AE, Gores GJ, Groopman JD, Selaru FM, Strazzabosco M, Wei Wang X, et al. Intrahepatic Cholangiocarcinoma: Continuing Challenges and Translational Advances. Hepatology. (2019) 69:1803-15. doi:10.1002/hep.30289

7 Albrecht TR, Shevtsov SP, Wu Y, Mascibroda LG, Peart NJ, Huang KL, et al. Integrator subunit 4 is a 'Symplekin-like' scaffold that associates with INTS9/11 to form the Integrator cleavage module. Nucleic Acids Res. (2018) 46:4241-55. doi:10.1093/nar/gky100

8 Kirstein N, Gomes Dos Santos H, Blumenthal E, and Shiekhattar R. The Integrator complex at the crossroad of coding and noncoding RNA. Curr Opin Cell Biol. (2020) 70:37-43. doi:10.1016/j.ceb.2020.11.003

9 Lai F, Gardini A, Zhang A, and Shiekhattar R. Integrator mediates the biogenesis of enhancer RNAs. Nature. (2015) 525:399-403. doi:10.1038/nature14906 
10 Huang KL, Jee D, Stein CB, Elrod ND, Henriques T, Mascibroda LG, et al. Integrator Recruits Protein Phosphatase 2A to Prevent Pause Release and Facilitate Transcription Termination. Mol Cell. (2020) 80:345-58.e9. doi:10.1016/j.molcel.2020.08.016

11 Zhang Y, Koe CT, Tan YS, Ho J, Tan P, Yu F, et al. The Integrator Complex Prevents Dedifferentiation of Intermediate Neural Progenitors back into Neural Stem Cells. Cell Rep. (2019) 27:987-96.e3. doi:10.1016/j.celrep.2019.03.089

12 Cheng L, Zhang Q, Yang S, Yang Y, Zhang W, Gao H, et al. A 4-gene panel as a marker at chromosome 8q in Asian gastric cancer patients. Genomics. (2013) 102:323-30. doi:10.1016/j.ygeno.2013.05.004

13 Wee Y, Wang T, Liu Y, Li X, and Zhao M. A pan-cancer study of copy number gain and up-regulation in human oncogenes. Life Sci. (2018) 211:206-14. doi:10.1016/j.1fs.2018.09.032

14 Tong H, Liu X, Li T, Qiu W, Peng C, Shen B, et al. INTS8 accelerates the epithelial-to-mesenchymal transition in hepatocellular carcinoma by upregulating the TGF- $\beta$ signaling pathway. Cancer Manag Res. (2019) 11:1869-79. doi:10.2147/cmar.S184392

15 Andersen JB, Spee B, Blechacz BR, Avital I, Komuta M, Barbour A, et al. Genomic and genetic characterization of cholangiocarcinoma identifies therapeutic targets for tyrosine kinase inhibitors. Gastroenterology. (2012) 142:1021-31.e15. doi:10.1053/j.gastro.2011.12.005

16 Sia D, Hoshida Y, Villanueva A, Roayaie S, Ferrer J, Tabak B, et al. Integrative molecular analysis of intrahepatic cholangiocarcinoma reveals 2 classes that have different outcomes. Gastroenterology. (2013) 144:829-40. doi:10.1053/j.gastro.2013.01.001

17 Leek JT, Johnson WE, Parker HS, Jaffe AE, and Storey JD. The sva package for removing batch effects and other unwanted variation in high-throughput experiments. Bioinformatics. 28:882-3. doi:10.1093/bioinformatics/bts034

18 Colaprico A, Silva TC, Olsen C, Garofano L, Cava C, Garolini D, et al. 
TCGAbiolinks: an R/Bioconductor package for integrative analysis of TCGA data. Nucleic Acids Res. (2016) 44:e71. doi:10.1093/nar/gkv1507

19 Wagner GP, Kin K, and Lynch VJ. Measurement of mRNA abundance using RNA-seq data: RPKM measure is inconsistent among samples. Theory Biosci. (2012) 131:281-5. doi:10.1007/s12064-012-0162-3

20 Law CW, Alhamdoosh M, Su S, Dong X, Tian L, Smyth GK, et al. RNA-seq analysis is easy as 1-2-3 with limma, Glimma and edgeR. F1000Res. (2016) 5. doi:10.12688/f1000research.9005.3

21 Kolde R, Laur S, Adler P, and Vilo J. Robust rank aggregation for gene list integration and meta-analysis. Bioinformatics. (2012) 28:573-80. doi:10.1093/bioinformatics/btr709

22 Mayakonda A, Lin DC, Assenov Y, Plass C, and Koeffler HP. Maftools: efficient and comprehensive analysis of somatic variants in cancer. Genome Res. (2018) 28:1747-56. doi:10.1101/gr.239244.118

23 Yu G, Wang LG, Han Y, and He QY. clusterProfiler: an R package for comparing biological themes among gene clusters. Omics. (2012) 16:284-7. doi:10.1089/omi.2011.0118

24 Becht E, Giraldo NA, Lacroix L, Buttard B, Elarouci N, Petitprez F, et al. Estimating the population abundance of tissue-infiltrating immune and stromal cell populations using gene expression. Genome Biol. (2016) 17:218. doi:10.1186/s13059-016-1070-5

25 Nusinow DP, Szpyt J, Ghandi M, Rose CM, Mcdonald ER, 3rd, Kalocsay M, et al. Quantitative Proteomics of the Cancer Cell Line Encyclopedia. Cell. (2020) 180:387-402.e16. doi:10.1016/j.cell.2019.12.023

26 Bahcall OG. Human genetics: GTEx pilot quantifies eQTL variation across tissues and individuals. Nat Rev Genet. (2015) 16:375. doi:10.1038/nrg3969

27 Jia D, Lin W, Tang H, Cheng Y, Xu K, He Y, et al. Integrative analysis of DNA methylation and gene expression to identify key epigenetic genes in glioblastoma. Aging (Albany NY). (2019) 11:5579-92. doi:10.18632/aging.102139 
28 Edwards JR, Yarychkivska O, Boulard M, and Bestor TH. DNA methylation and DNA methyltransferases. Epigenetics Chromatin. (2017) 10:23. doi:10.1186/s13072-017-0130-8

29 Kelley RK, Bridgewater J, Gores GJ, and Zhu AX. Systemic therapies for intrahepatic cholangiocarcinoma. $J$ Hepatol. (2020) 72:353-63. doi:10.1016/j.jhep.2019.10.009

30 Federico A, Rienzo M, Abbondanza C, Costa V, Ciccodicola A, and Casamassimi A. Pan-Cancer Mutational and Transcriptional Analysis of the Integrator Complex. Int J Mol Sci. (2017) 18. doi:10.3390/ijms18050936

31 Liu Z, Sun Y, Jiang Y, Qian Y, Chen S, Gao S, et al. Follicle-stimulating hormone (FSH) promotes retinol uptake and metabolism in the mouse ovary. Reprod Biol Endocrinol. (2018) 16:52. doi:10.1186/s12958-018-0371-9

32 Marceau G, Gallot D, Lemery D, and Sapin V. Metabolism of retinol during mammalian placental and embryonic development. Vitam Horm. (2007) 75:97115. doi:10.1016/s0083-6729(06)75004-X

33 Romeo S, and Valenti L. Regulation of retinol-binding protein 4 and retinol metabolism in fatty liver disease. Hepatology. (2016) 64:1414-16. doi:10.1002/hep.28722

34 Ohkawa K, Abe T, Hatano T, Takizawa N, Yamada K, and Takada K. The facilitated effect of retinol on rat hepatocarcinogenesis induced by 3'-methyl-4dimethylaminoazobenzene. Carcinogenesis. (1991) 12:2357-60. doi:10.1093/carcin/12.12.2357

35 Müller CM, Scierka A, Stiller RL, Kim YM, Cook DR, Lancaster JR, Jr., et al. Nitric oxide mediates hepatic cytochrome P450 dysfunction induced by endotoxin. Anesthesiology. (1996) 84:1435-42. doi:10.1097/00000542199606000-00020

$36 \mathrm{Xu} \mathrm{N}, \mathrm{Wu}$ YP, Ke ZB, Liang YC, Cai H, Su WT, et al. Identification of key DNA methylation-driven genes in prostate adenocarcinoma: an integrative analysis of TCGA methylation data. J Transl Med. (2019) 17:311. doi:10.1186/s12967-019-2065-2 
37 Yongvanit P, Phanomsri E, Namwat N, Kampan J, Tassaneeyakul W, Loilome W, et al. Hepatic cytochrome P450 2A6 and 2E1 status in peri-tumor tissues of patients with Opisthorchis viverrini-associated cholangiocarcinoma. Parasitol Int. (2012) 61:162-6. doi:10.1016/j.parint.2011.06.026

38 Currie E, Schulze A, Zechner R, Walther TC, and Farese RV, Jr. Cellular fatty acid metabolism and cancer. Cell Metab. (2013) 18:153-61. doi:10.1016/j.cmet.2013.05.017

39 Cheng C, Geng F, Cheng X, and Guo D. Lipid metabolism reprogramming and its potential targets in cancer. Cancer Commun (Lond). (2018) 38:27. doi:10.1186/s40880-018-0301-4

40 Li L, Che L, Tharp KM, Park HM, Pilo MG, Cao D, et al. Differential requirement for de novo lipogenesis in cholangiocarcinoma and hepatocellular carcinoma of mice and humans. Hepatology. (2016) 63:1900-13. doi:10.1002/hep. 28508

41 Williams AB, and Schumacher B. p53 in the DNA-Damage-Repair Process. Cold Spring Harb Perspect Med. (2016) 6. doi:10.1101/cshperspect.a026070

42 Phillips DH. Mutational spectra and mutational signatures: Insights into cancer aetiology and mechanisms of DNA damage and repair. DNA Repair (Amst). (2018) 71:6-11. doi:10.1016/j.dnarep.2018.08.003

43 Yang Y, Li X, Hao L, Jiang D, Wu B, He T, et al. The diagnostic value of DNA repair gene in breast cancer metastasis. Sci Rep. (2020) 10:19626. doi:10.1038/s41598-020-76577-2

44 Liengswangwong U, Karalak A, Morishita Y, Noguchi M, Khuhaprema T, Srivatanakul P, et al. Immunohistochemical expression of mismatch repair genes: a screening tool for predicting mutator phenotype in liver fluke infectionassociated intrahepatic cholangiocarcinoma. World J Gastroenterol. (2006) 12:3740-5. doi:10.3748/wjg.v12.i23.3740

45 Sato Y, Tanaka S, Kinoshita M, Takemura S, Shinkawa H, Kokudo T, et al. Immunosuppressive tumor microenvironment in occupational cholangiocarcinoma: Supportive evidence for the efficacy of immune 
checkpoint inhibitor therapy. J Hepatobiliary Pancreat Sci. (2020) 27:860-69. doi:10.1002/jhbp.788

46 Winkelmann R, Schneider M, Hartmann S, Schnitzbauer AA, Zeuzem S, Peveling-Oberhag J, et al. Microsatellite Instability Occurs Rarely in Patients with Cholangiocarcinoma: A Retrospective Study from a German Tertiary Care Hospital. Int J Mol Sci. (2018) 19. doi:10.3390/ijms19051421

47 Parsons MT, Buchanan DD, Thompson B, Young JP, and Spurdle AB. Correlation of tumour BRAF mutations and MLH1 methylation with germline mismatch repair (MMR) gene mutation status: a literature review assessing utility of tumour features for MMR variant classification. J Med Genet. (2012) 49:151-7. doi:10.1136/jmedgenet-2011-100714

48 Ju JY, Dibbern ME, Mahadevan MS, Fan J, Kunk PR, and Stelow EB. Mismatch Repair Protein Deficiency/Microsatellite Instability Is Rare in Cholangiocarcinomas and Associated With Distinctive Morphologies. Am J Clin Pathol. (2020) 153:598-604. doi:10.1093/ajcp/aqz199

49 Abraham SC, Lee JH, Boitnott JK, Argani P, Furth EE, and Wu TT. Microsatellite instability in intraductal papillary neoplasms of the biliary tract. Mod Pathol. (2002) 15:1309-17. doi:10.1097/01.Mp.0000038461.80167.34

50 Kim GJ, Kim H, and Park YN. Increased expression of Yes-associated protein 1 in hepatocellular carcinoma with stemness and combined hepatocellularcholangiocarcinoma. PLoS One. (2013) 8:e75449. doi:10.1371/journal.pone.0075449

51 Wakizaka K, Yokoo H, Kamiyama T, Kakisaka T, Ohira M, Tani M, et al. CD133 and epithelial cell adhesion molecule expressions in the cholangiocarcinoma component are prognostic factors for combined hepatocellular cholangiocarcinoma. Hepatol Res. (2020) 50:258-67. doi:10.1111/hepr.13443

52 Jones PA, and Baylin SB. The epigenomics of cancer. Cell. (2007) 128:683-92. doi:10.1016/j.cell.2007.01.029

53 Ginder GD, and Williams DC, Jr. Readers of DNA methylation, the MBD 
family as potential therapeutic targets. Pharmacol Ther. (2018) 184:98-111. doi:10.1016/j.pharmthera.2017.11.002

54 Baylin SB, and Jones PA. Epigenetic Determinants of Cancer. Cold Spring Harb Perspect Biol. (2016) 8. doi:10.1101/cshperspect.a019505

55 Lyko F. The DNA methyltransferase family: a versatile toolkit for epigenetic regulation. Nat Rev Genet. (2018) 19:81-92. doi:10.1038/nrg.2017.80

56 Tamma R, Annese T, Ruggieri S, Brunetti O, Longo V, Cascardi E, et al. Inflammatory cells infiltrate and angiogenesis in locally advanced and metastatic cholangiocarcinoma. Eur J Clin Invest. (2019) 49:e13087. doi:10.1111/eci.13087

57 Alnaggar M, Xu Y, Li J, He J, Chen J, Li M, et al. Allogenic V $\gamma 9 \mathrm{~V} \delta 2$ T cell as new potential immunotherapy drug for solid tumor: a case study for cholangiocarcinoma. J Immunother Cancer. (2019) 7:36. doi:10.1186/s40425019-0501-8

58 Zhang M, Yang H, Wan L, Wang Z, Wang H, Ge C, et al. Single-cell transcriptomic architecture and intercellular crosstalk of human intrahepatic cholangiocarcinoma. $\quad J \quad$ Hepatol. (2020) 73:1118-30. doi:10.1016/j.jhep.2020.05.039

\section{Acknowledgements}

We sincerely thank the TCGA and GEO database for providing their platforms and contributors for their valuable datasets. This research was supported by the Leading Talent of Hundred, Thousand and Ten Thousand Project of Xingliao Gifted Person Program of Liaoning Province (NO. XLYC1905013), the Leading Talent Team of Support Program for High-Level Talent's Innovation of Dalian in 2019, and the Type A Project of Leading Talent's Innovative Research of Dalian.

\section{Ethical approval statement}

Informed consent was obtained from all participants. The study involving human participants was approved by the Ethics Committee of Shanghai Outdo Biotech 
Company with NO. YB M-05-02, and performed following relevant guidelines and regulations.

\section{Author contributions}

Dong Shang and Shilin Xia contributed to designing the study and revising the manuscript. Qi Zhou, Li Ji and Xueying Shi performed the experiments and drafted the manuscript. Dawei Deng, Fangyue Guo and Zhengpeng Wang provided the method support and coordinated the study. Wenhui Liu and Jinnan Zhang contributed to the figures and tables. All the authors reviewed and approved the final manuscript.

\section{Conflict of interest}

The authors report no conflict of interest concerning the materials or methods used in this study or the findings specified in this paper.

Table 1. Correlation between INTS8 expression and clinicopathological characteristics.

\begin{tabular}{|c|c|c|c|c|c|c|}
\hline & \multirow{2}{*}{ variables } & \multicolumn{2}{|c|}{ INTS8 expression } & \multirow{2}{*}{ total } & \multirow{2}{*}{$x^{2}$} & \multirow{2}{*}{$\mathrm{p}$ value } \\
\hline & & low & high & & & \\
\hline \multirow[t]{3}{*}{ Age (year) } & & & & & 4.123 & 0.042 \\
\hline & $<60$ & 36 & 27 & 63 & & \\
\hline & $\geq 60$ & 36 & 53 & 89 & & \\
\hline \multirow[t]{3}{*}{ Sex } & & & & & 0.553 & 0.457 \\
\hline & Female & 30 & 29 & 59 & & \\
\hline & male & 42 & 52 & 94 & & \\
\hline \multirow[t]{3}{*}{ Grade } & & & & & 15.516 & 0.000 \\
\hline & $\mathrm{I} / \mathrm{II}$ & 60 & 45 & 105 & & \\
\hline & III & 11 & 37 & 48 & & \\
\hline \multirow[t]{3}{*}{ T stage } & & & & & 0.048 & 0.773 \\
\hline & $\mathrm{T} 1$ & 1 & 2 & 3 & & \\
\hline & $\mathrm{T} 2 / \mathrm{T} 4$ & 13 & 18 & 31 & & \\
\hline \multirow[t]{3}{*}{ N stage } & & & & & 0.192 & 0.661 \\
\hline & No & 20 & 23 & 40 & & \\
\hline & N1 & 12 & 11 & 23 & & \\
\hline \multirow[t]{3}{*}{ M stage } & & & & & 0.045 & 0.833 \\
\hline & M0 & 69 & 78 & 147 & & \\
\hline & M1 & 3 & 4 & 7 & & \\
\hline \multirow[t]{3}{*}{ TNM stage } & & & & & 0.026 & 0.871 \\
\hline & $\mathrm{I} / \mathrm{II}$ & 10 & 11 & 21 & & \\
\hline & IV & 13 & 13 & 26 & & \\
\hline
\end{tabular}


Tumor size

\begin{tabular}{llll}
$\geq 7$ & 33 & 42 & 75 \\
$<7$ & 32 & 33 & 65 \\
\hline
\end{tabular}

$*$ Statistically significant $(\mathrm{p}<0.05)$

Figure Legends

Figure 1. The identification of RRA gene set. (A-D) Differentially expressed genes of 2 GSE datasets. (E) The visualization of RRA gene set.

Figure 2. Mutation landscape of RRA gene set in TCGA-CHOL. (A-C) According to different classification categories, classification of mutation types including missense mutation, SNP, and C $>$ T mutation were showed with statistical calculations. (D) The total mutation number in each sample. (E) Each variant classification in each sample. (F) The top 10 mutated genes in TCGA-CHOL. (G)The mutation information of 5 mutation genes in RRA gene set performed by the waterfall plot. (H) The top mutant genes of RRA gene set showed by box plot. (I) The mutant base pair ratio of RRA gene set.

Figure 3. The identification of INTS8 serving as the candidate gene. (A) The ROC curves of 5 genes for diagnosis value. (B) The DEGs in high and low- INTS8 expression groups. (C) The expression of INTS8 in a human normal biliary epithelial cell line (HIBE) and 3 ICC cell lines (including HCCC-9810, RBE, and CCLP-1 cells) by using PCR. (D) Representative images of INTS8 IHC staining in human ICC and adjacent normal tissues.

Figure 4. Functional enrichment of INTS8 related genes in CHOL. (A-B) GO and KEGG analyses of INTS8 related genes. (C-D) GSEA-GO and GSEA-KEGG analyses of INTS8 related genes.

Figure 5. The identification of complex associations between 22 TIC subsets and INTS8 expression in CHOL. (A) The relative proportions of 22 subtypes of tumorinfiltrating immune cells for each sample in CHOL. (B) The relative proportions of 22 
subtypes of tumor-infiltrating immune cells for each sample. (C-D) The comparison of the immune cell fraction difference between low and high INTS8 expression groups.

Figure 6. The INTS8 expression in multiple dimensions. Expression analysis of INTS8 gene in CCLE (A) and GTEx (B) and the somatic mutation profile of INTS8 gene in TCGA database (C).

Figure 7. The MMRs and DNA methylation involved in CHOL. (A) A total of 3 MMRs were detected in CHOL. (B) Circle diagram showed the association between INTS8 and DNA methyltransferases. 
Figures

A

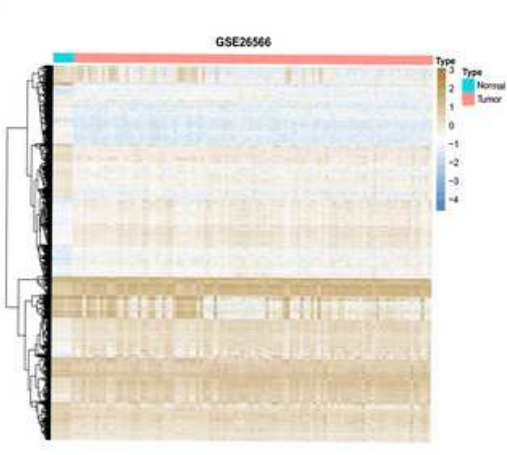

C

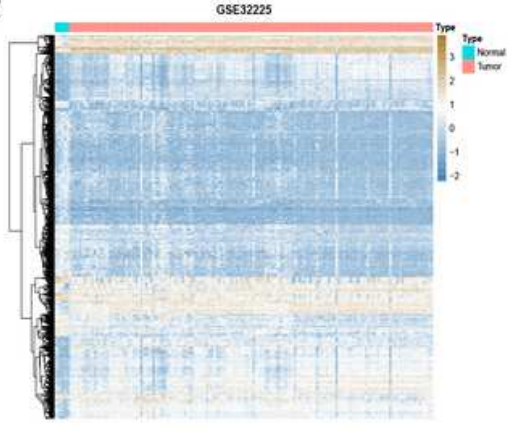

B

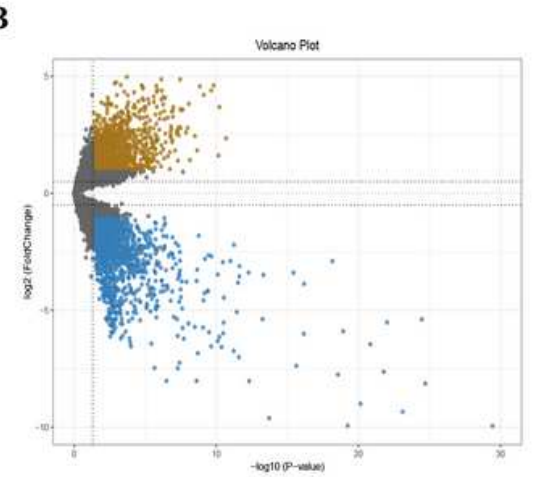

D

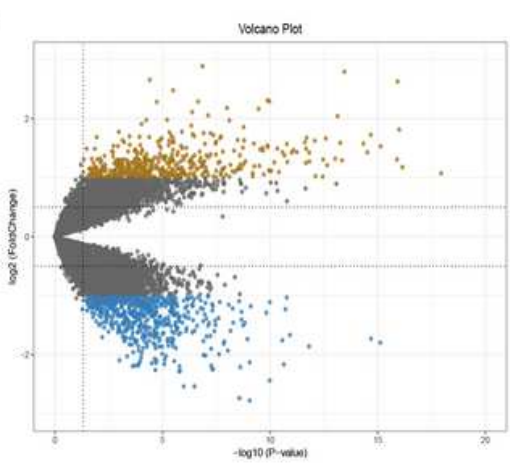

E

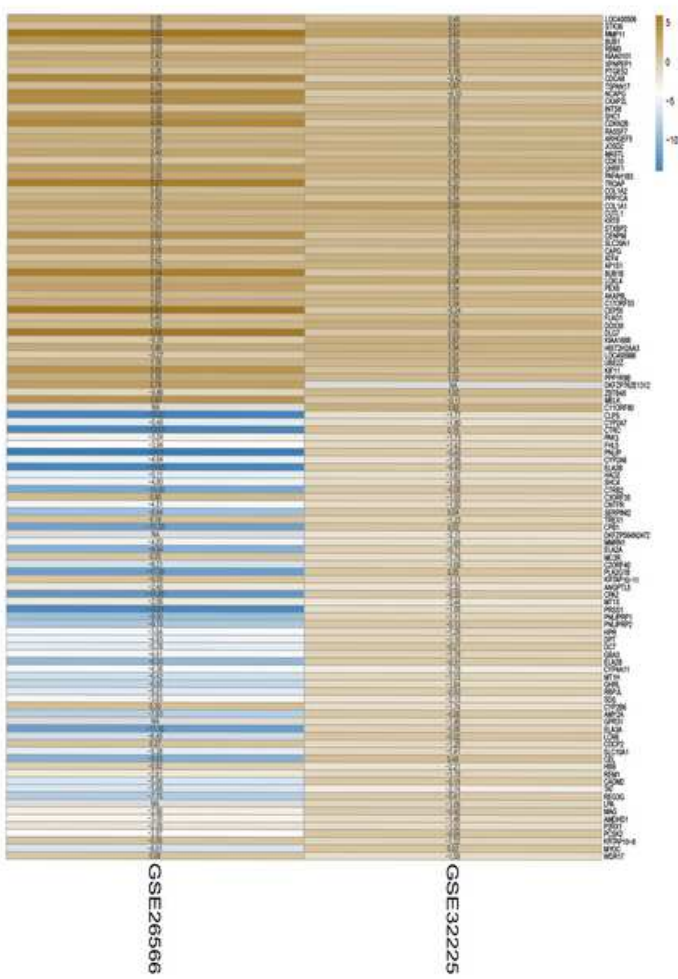

Figure 1

The identification of RRA gene set. (A-D) Differentially expressed genes of 2 GSE datasets. (E) The visualization of RRA gene set. 
A

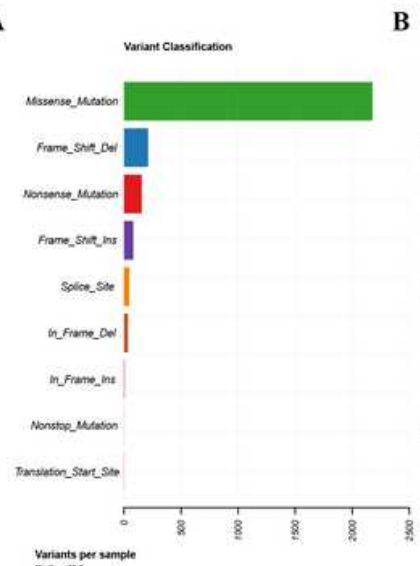

B

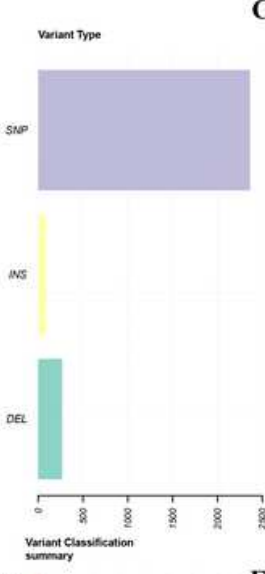

C swe cluss
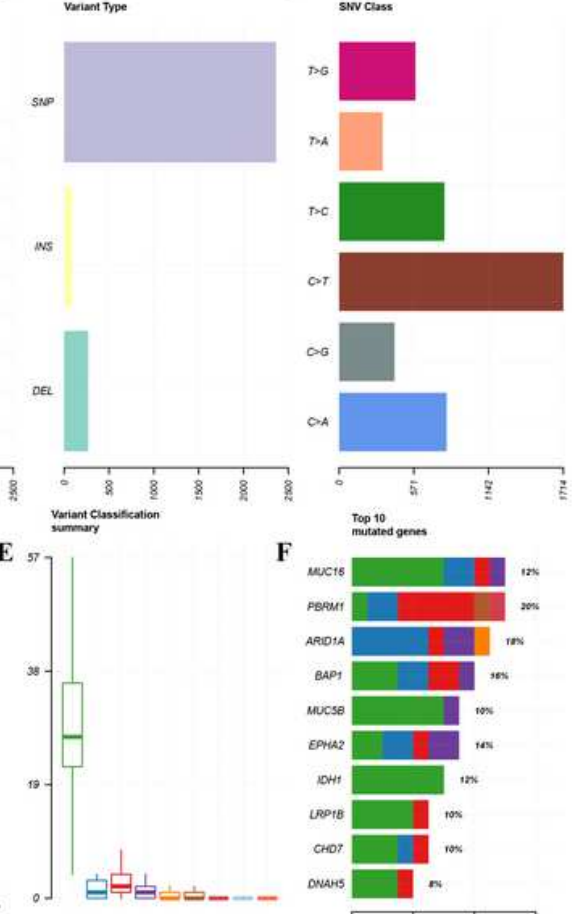

F

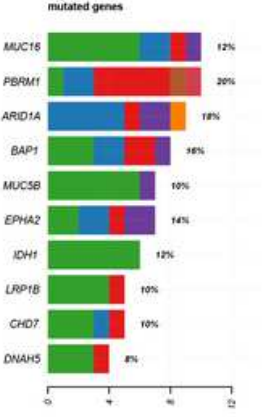

G
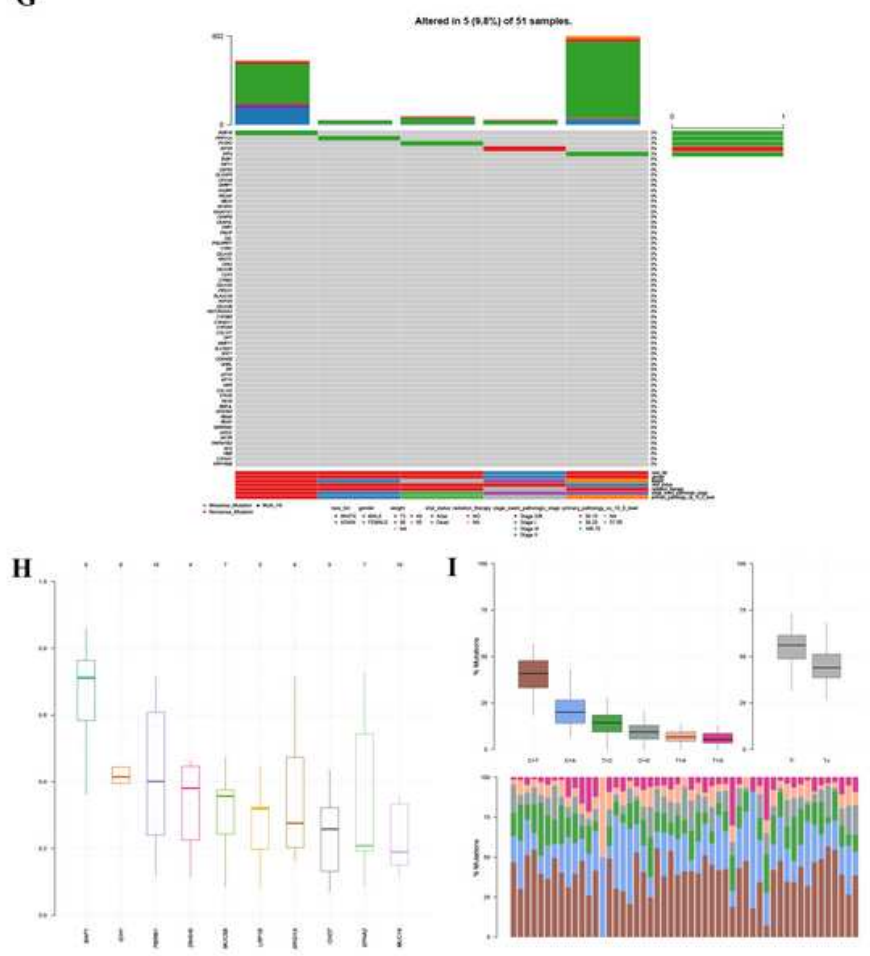

Figure 2

Mutation landscape of RRA gene set in TCGA-CHOL. (A-C) According to different classification categories, classification of mutation types including missense mutation, SNP, and $C>T$ mutation were showed with statistical calculations. (D) The total mutation number in each sample. (E) Each variant classification in each sample. (F) The top 10 mutated genes in TCGA-CHOL. (G)The mutation information of 5 mutation genes in RRA gene set performed by the waterfall plot. $(\mathrm{H})$ The top mutant genes of RRA gene set showed by box plot. (I) The mutant base pair ratio of RRA gene set. 
A

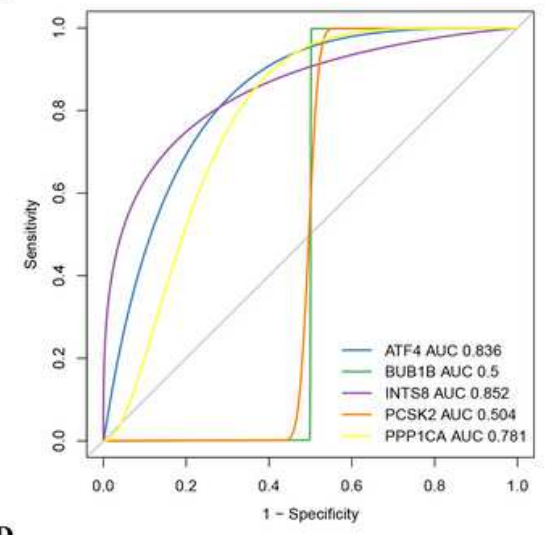

D
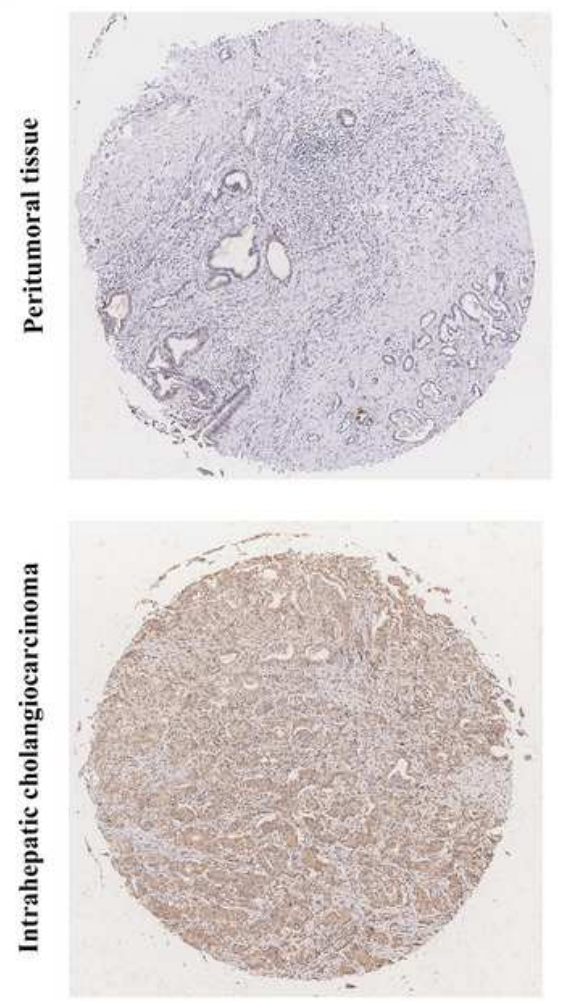

B

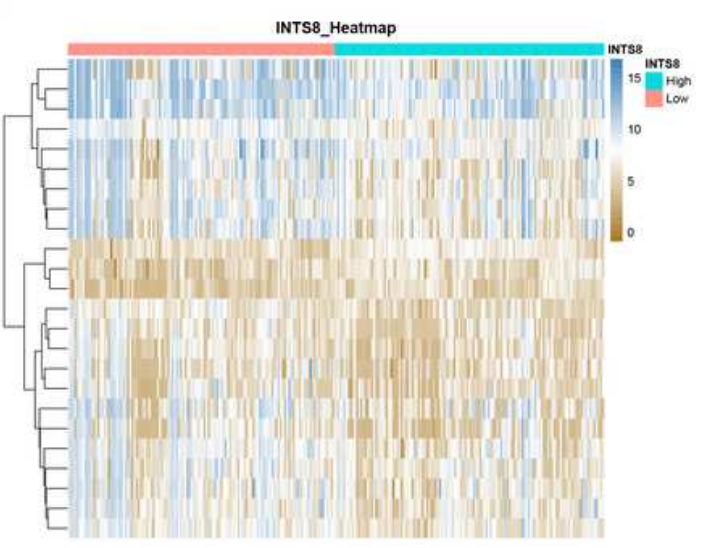

C

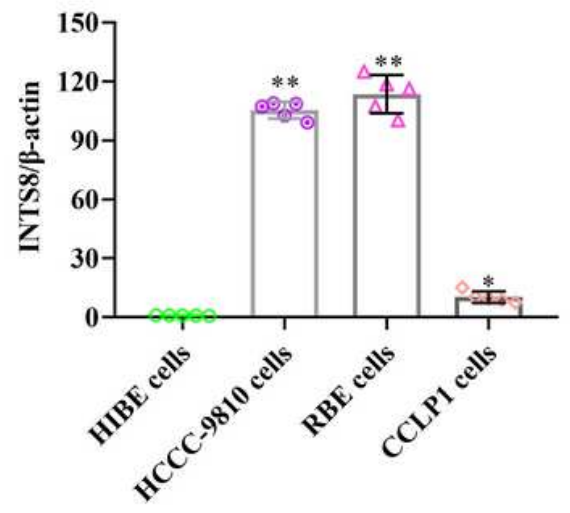

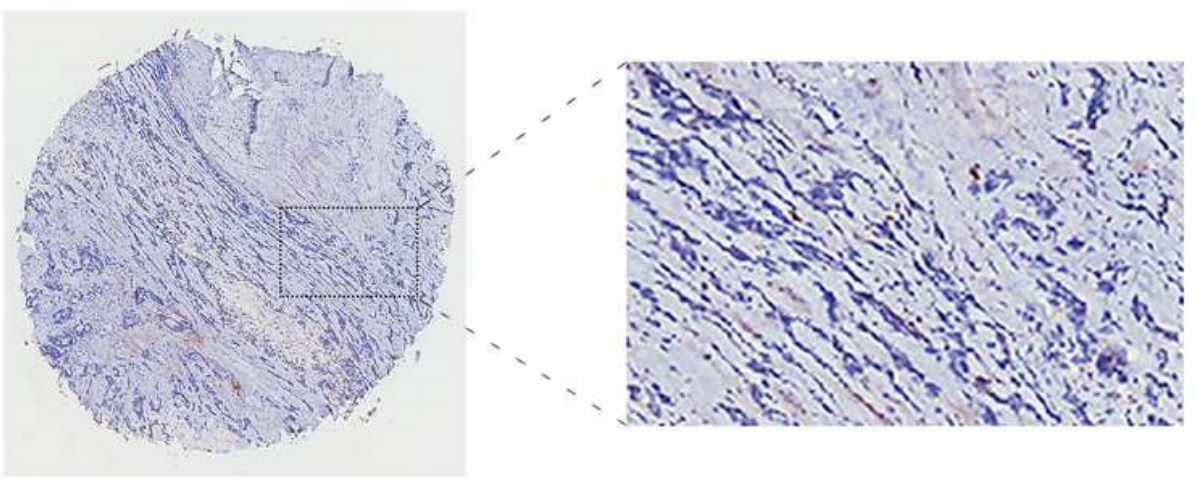

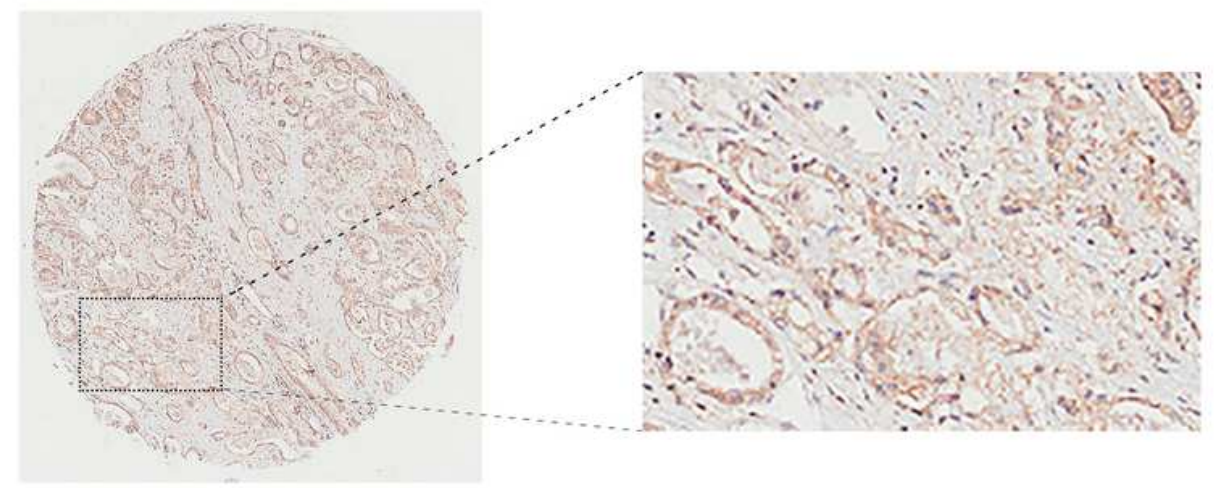

\section{Figure 3}

The identification of INTS8 serving as the candidate gene. (A) The ROC curves of 5 genes for diagnosis value. (B) The DEGs in high and low- INTS8 expression groups. (C) The expression of INTS8 in a human normal biliary epithelial cell line (HIBE) and 3 ICC cell lines (including HCCC-9810, RBE, and CCLP-1 cells) by using PCR. (D) Representative images of INTS8 IHC staining in human ICC and adjacent normal tissues 
A

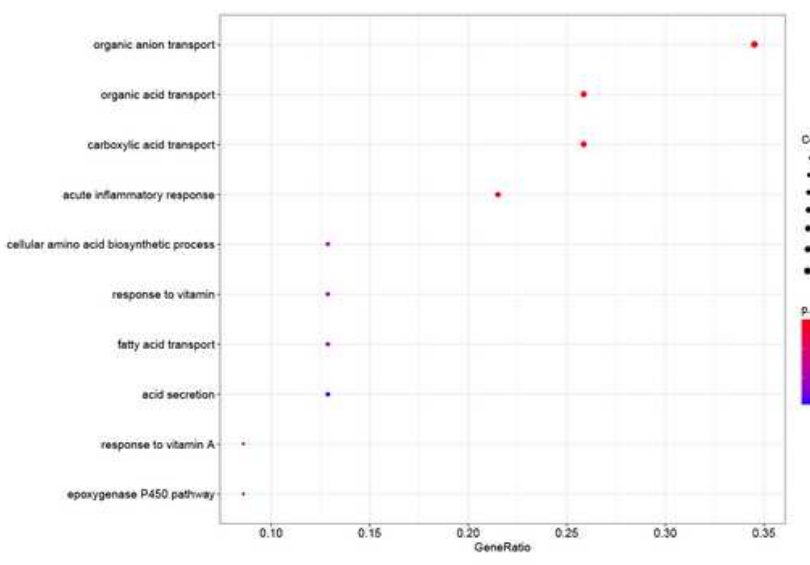

C

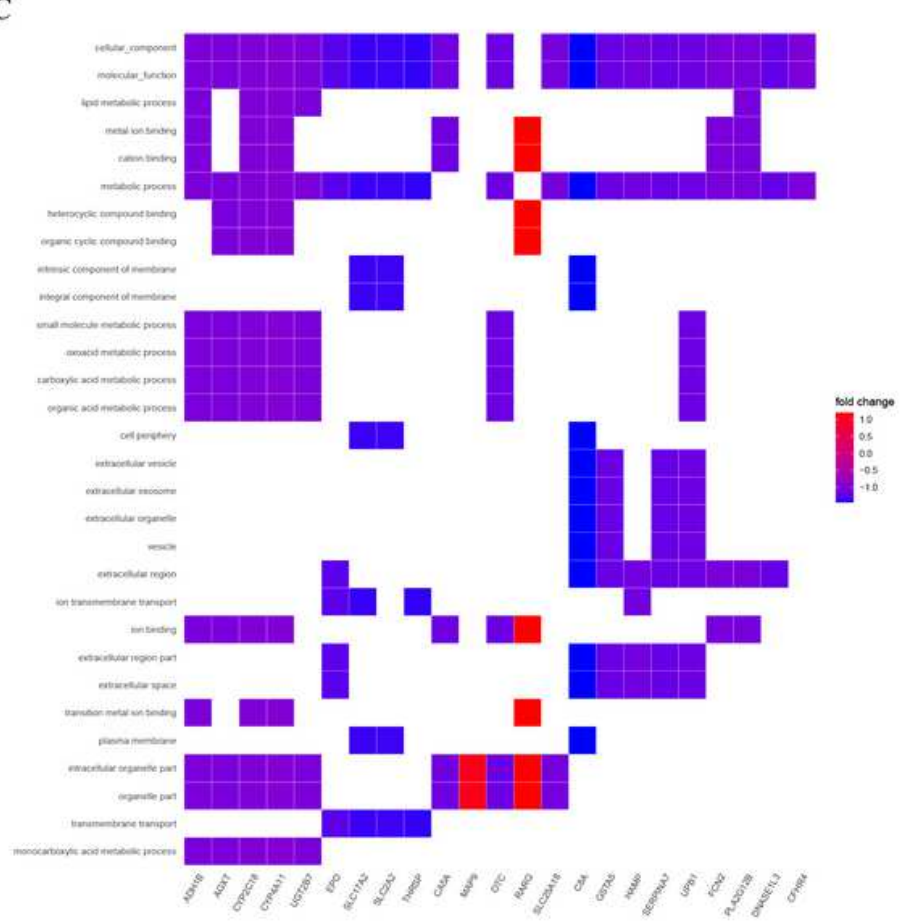

B

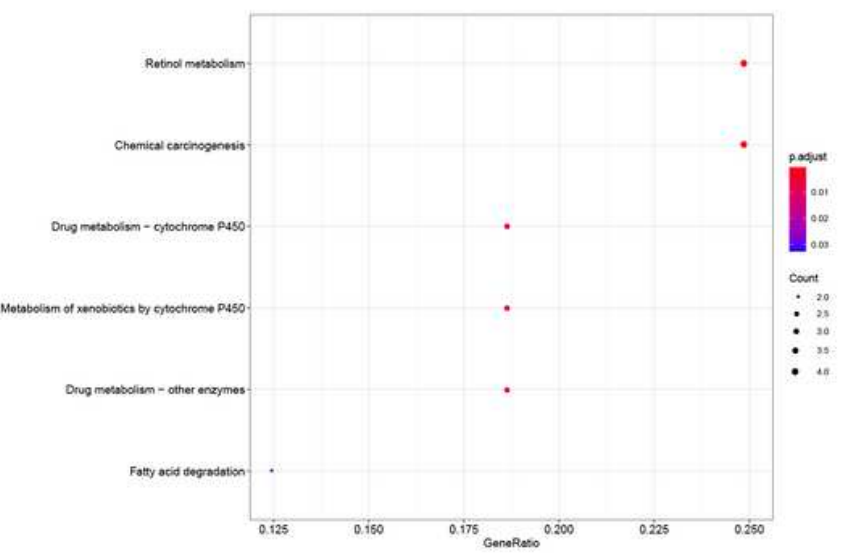

D

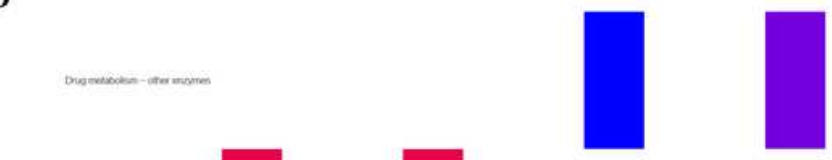

Figure 4

Functional enrichment of INTS8 related genes in CHOL. (A-B) GO and KEGG analyses of INTS8 related genes. (C-D) GSEA-GO and GSEA-KEGG analyses of INTS8 related genes. 
A
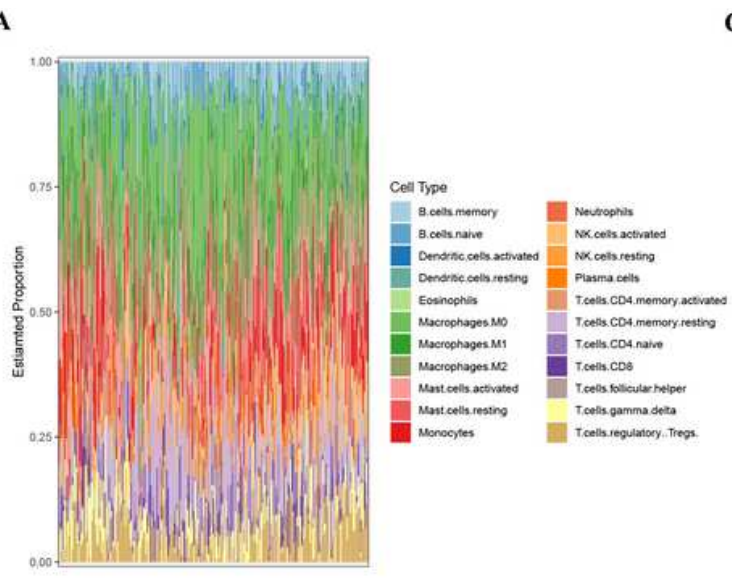

B

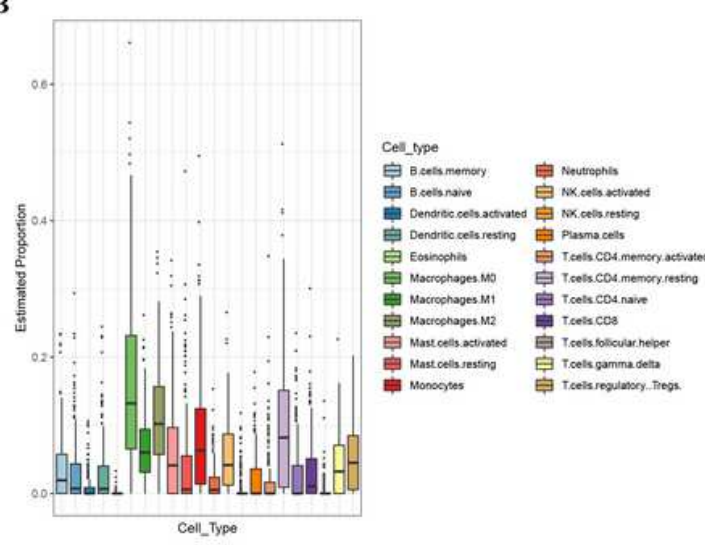

D
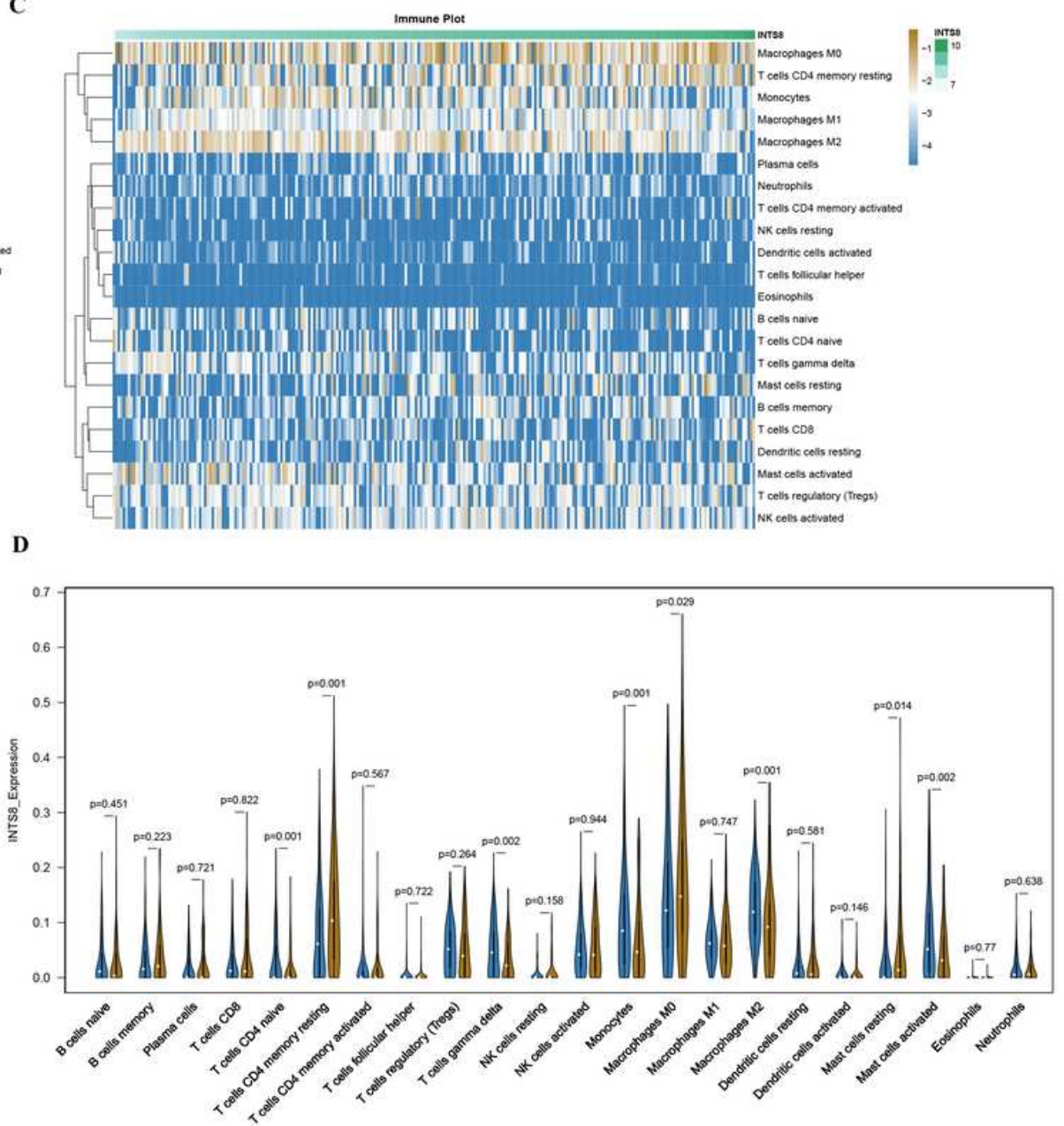

Figure 5

The identification of complex associations between 22 TIC subsets and INTS8 expression in CHOL. (A) The relative proportions of 22 subtypes of tumorखinfiltrating immune cells for each sample in CHOL. (B) The relative proportions of 22 subtypes of tumor-infiltrating immune cells for each sample. (C-D) The comparison of the immune cell fraction difference between low and high INTS8 expression groups. 
A

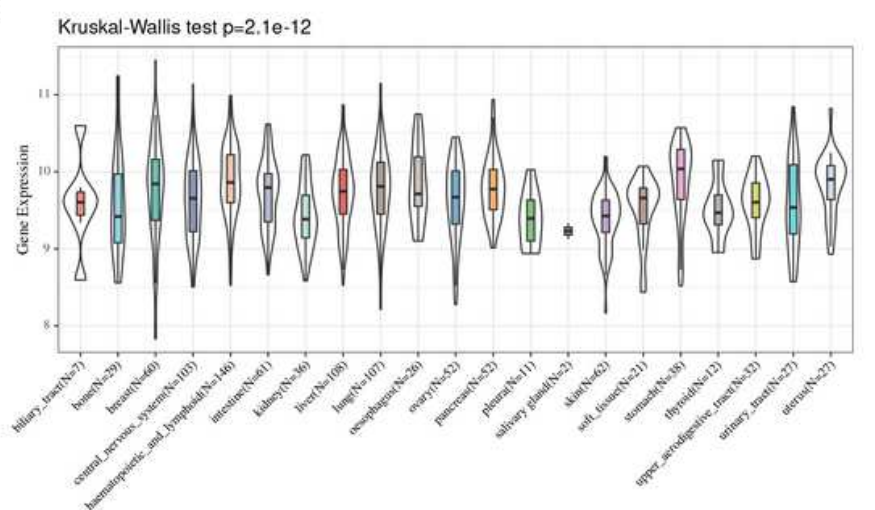

B

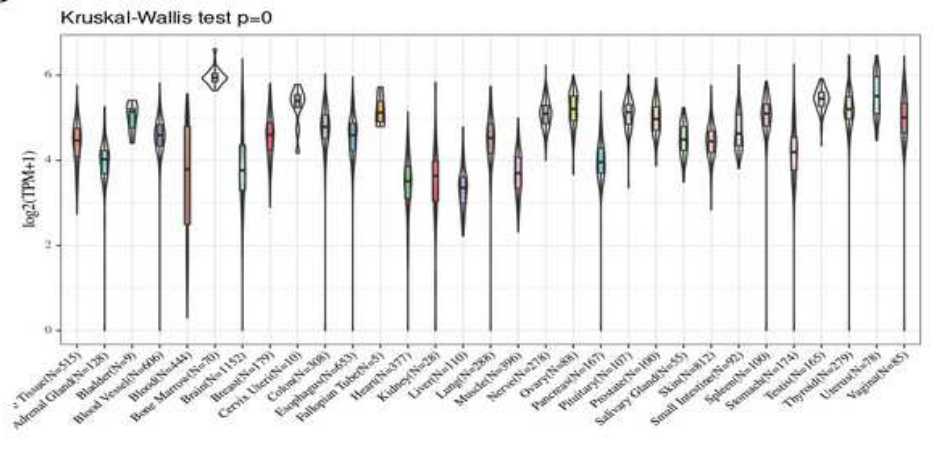

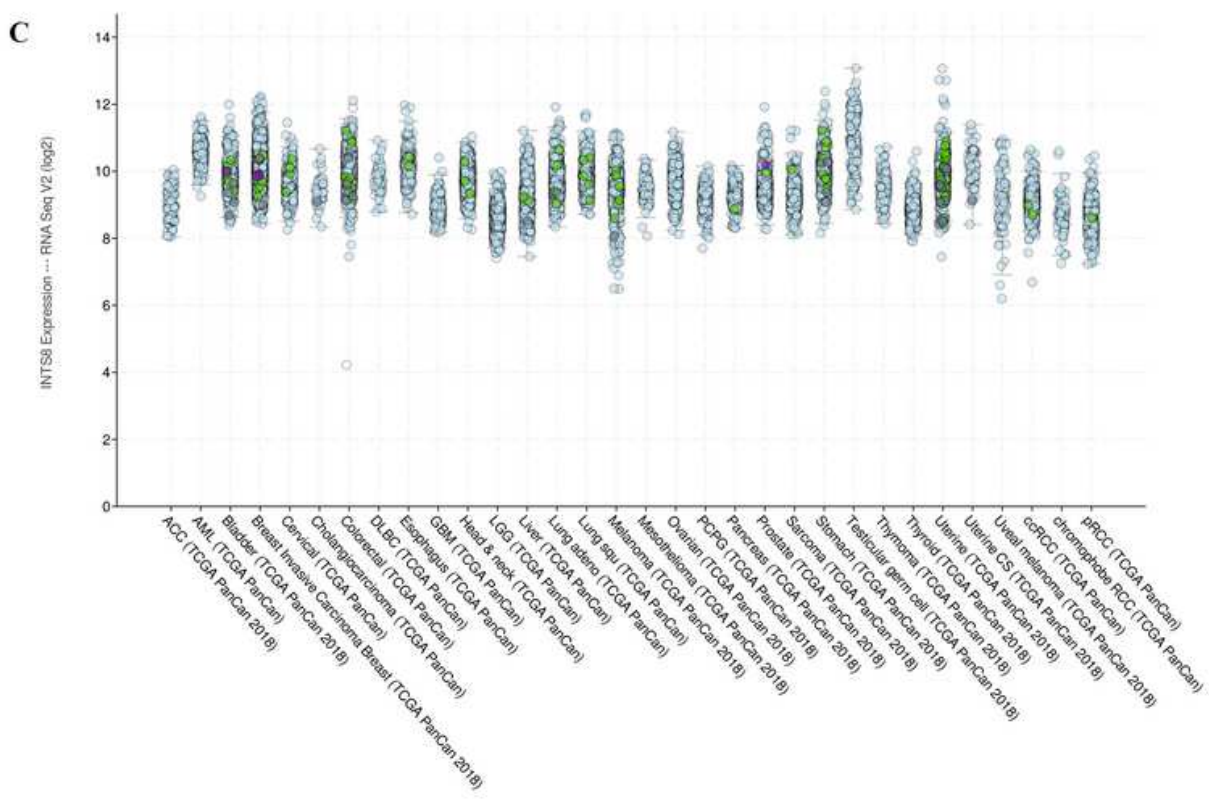

$\begin{array}{ll}\text { - Fusion } & \text { - Truncating (VUS) } \\ \text { - Not mutated } & \text { Not profiled for mutations }\end{array}$

- Intrame (VUS) - Missense (VUS)

\section{Figure 6}

The INTS8 expression in multiple dimensions. Expression analysis of INTS8 gene in CCLE (A) and GTEx (B) and the somatic mutation profile of INTS8 gene in TCGA database (C). 
A

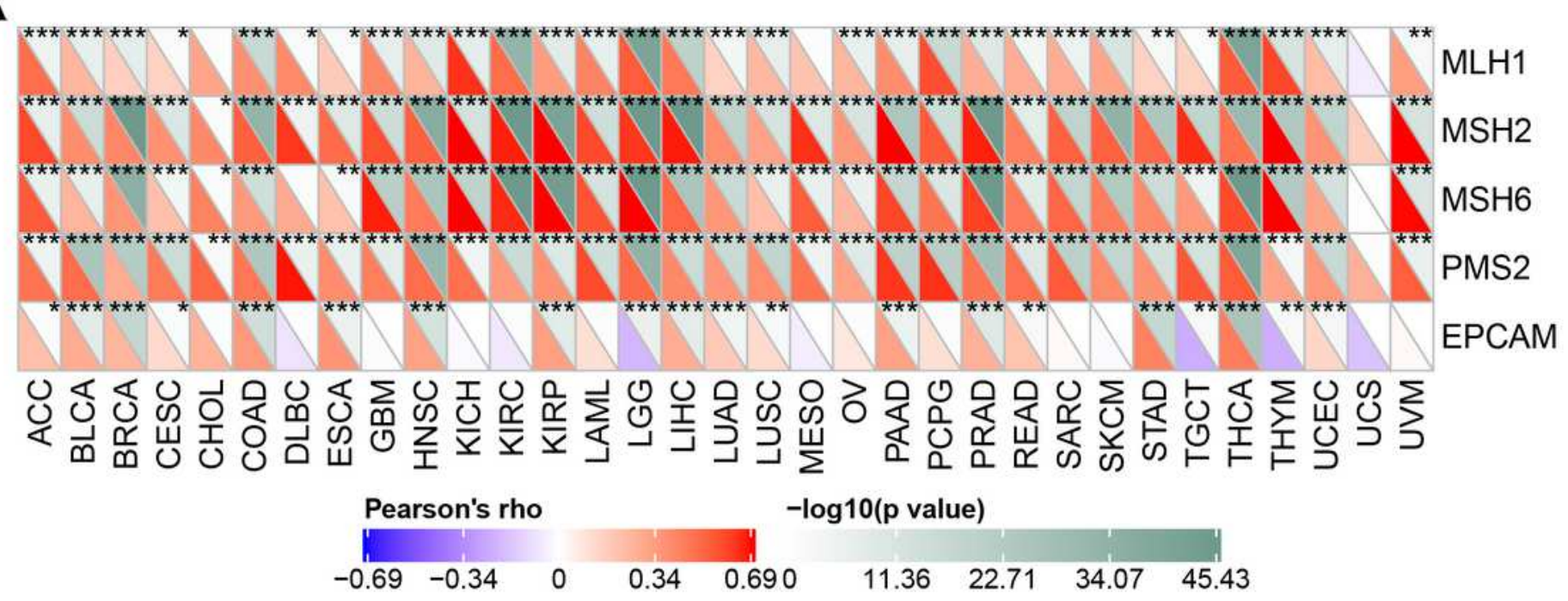

B

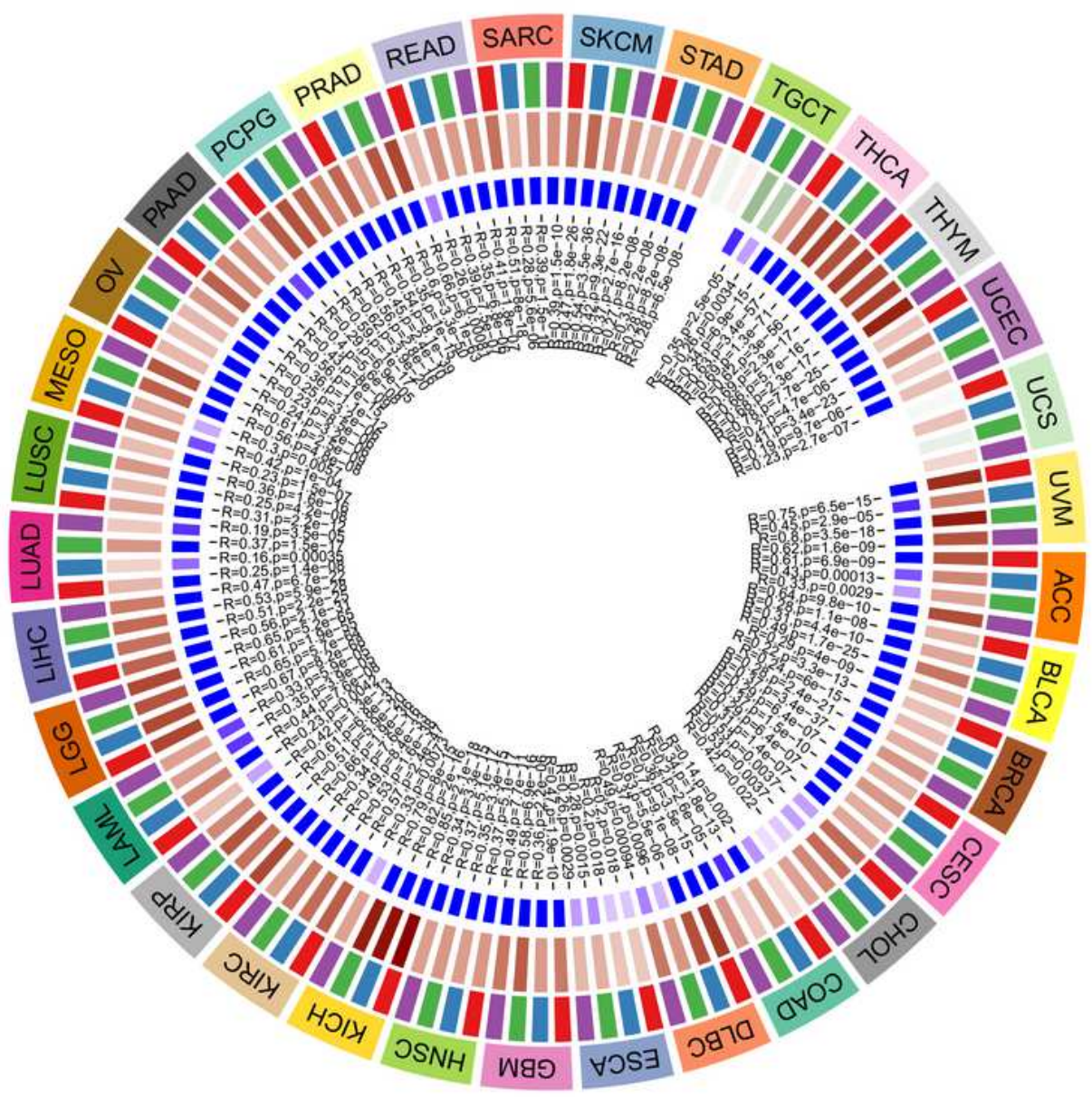

Figure 7

The MMRs and DNA methylation involved in CHOL. (A) A total of 3 MMRs were detected in CHOL. (B) Circle diagram showed the association between INTS8 and DNA methyltransferases

\section{Supplementary Files}


This is a list of supplementary files associated with this preprint. Click to download.

- SupplementaryMaterial.pdf 